\title{
Pregabalin-induced first degree atrioventricular block in a young patient treated for pain from extrapulmonary tuberculosis
}

\author{
Alessandra Schiavo1*, Francesca M. Stagnaro1*, Andrea Salzano1,2, Alberto M. Marra3, \\ Emanuele Bobbio1, Pietro Valente1, Simona Grassi', Martina Miniero1, Michele Arcopinto1, \\ Margherita Matarazzo', Raffaele Napoli1, Antonio Cittadini1 \\ * These authors contributed equally \\ 1 Department of Translational Medical Sciences, Federico II University School of Medicine, Naples, Italy \\ 2 Department of Cardiovascular Sciences and NIHR Leicester Biomedical Research Centre, University of \\ Leicester, Glenfield Hospital, Groby Road, Leicester, UK \\ 3 IRCCS SDN, Naples, Italy
}

\begin{abstract}
Pregabalin, widely used in the treatment of several pain disorders, is usually well tolerated. Uncommonly, the drug may induce cardiac side effects, rarely prolongation of the PR interval. The latter has never been described in patients with healthy heart or normal renal function. We characterize a unique case of a young man with extrapulmonary tuberculosis and no detectable or known cardiac or kidney diseases, treated with pregabalin to control the severe pain due to the involvement of the spinal cord by the tuberculosis, showing an atrioventricular (AV) block due to pregabalin administration. The reported case emphasizes the need of monitoring PR interval during treatment with pregabalin, even in patients without background of cardiac or renal diseases.
\end{abstract}

Corresponding author: Antonio Cittadini, Department of Translational Medical Sciences, Federico II University School of Medicine, Via Pansini 5, 80131 Napoli, Italy. Tel/Fax +39.081 .7464375 .

E-mail: antonio.cittadini@unina.it

Key words: Pregabalin; atrioventricular block; cardiovascular side effects; tuberculosis; pain.

Acknowledgments: We thank all clinical staff of the hospital for its dedication to the patient wellbeing.

Contributions: ASc, FS, RN, AC, manuscript writing, data collection; ASc, FS, $\mathrm{PV}$, radiological imagine collection; AMM, ASa, EB, SG, MMi, MA, manuscript writing; MA, MMa, RN, AC, case supervision. All authors contributed to the interpretation of the data, read and approved the final manuscript.

Conflict of interest: the authors declare no conflict of interest.

Received for publication: 18 May 2017

Accepted for publication: 20 September 2017

(C) Copyright A. Schiavo et al., 2017

Tipografia PI-ME Editrice, Italy

Monaldi Archives for Chest Disease 2017; 87:838

doi: 10.4081/monaldi.2017.838

This article is distributed under the terms of the Creative Commons Attribution Noncommercial License (by-nc 4.0) which permits any noncommercial use, distribution, and reproduction in any medium, provided the original author(s) and source are credited.

\section{Introduction}

Pregabalin is widely used in the treatment of several pain disorders, including pain due to peripheral neuropathy, i.e. diabetic neuropathy, spinal cord injury, post-herpetic neuralgia, fibromyalgia. In addition, it exerts antiepileptic, analgesic, and anticonvulsant activity. The drug is a synthetic analogue of the inhibitory neurotransmitter $\gamma$-aminobutyric acid (GABA) and acts by reducing the release of several neurotransmitters (glutamate, norepinephrine, serotonin, dopamine and substance P) through the binding to the $\alpha 2-\delta$ subunit of presynaptic, voltage-dependent calcium channels [1]. Generally, very well-tolerated, pregabalin may occasionally induce some adverse events, that are mild to moderate in nature and usually rapidly transient. More frequently the drug might induce somnolence and dizziness, whereas, less frequently, peripheral edema, weight gain, headache, dry mouth, and ataxia [1]. In contrast, cardiovascular side effects are uncommon with an estimated occurrence being between $>1 / 1000$ and $<1 / 100$ cases. Among them, tachycardia is more prevalent, whereas prolongation of the PR interval recorded at the electrocardiogram (EKG) is considered quite unusual. In addition, such an event has been reported only in conjunction with either impaired renal function or heart disease. Now, we present the unexpected case of a young black man, without known or detectable cardiovascular or kidney diseases, who incurred in a $1^{\text {st }}$ degree atrioventricular (AV) block during the treatment with pregabalin.

\section{Case Report}

A 20-year-old black man, complaining of intense back pain radiating down the legs, was admitted to our internal medicine unit. Such pain was described as particularly intense, steady, burning, and interfering with the sleep. The patient portrayed a progressive weight loss of $15 \mathrm{Kg}$ in the last 10 months. Before accessing our unit, he had been examined by CT scan showing two voluminous masses, one involving the spinal cord (L4-L5/L5-S1) and the sacral bone (Figure 1A), the other the sternum (Figure 1B). At physical examination, two large subcutaneous masses (about $10 \mathrm{~cm}$ ) were observed in the left parasternal area, one of which was ulcerated and pouring a necrotic and suppurative leak. Systemic arterial blood pressure was $110 / 80 \mathrm{mmHg}$ with a pulse rate of $90 \mathrm{bpm}$. With the exception of a slight lymphopenia $\left(0.57 \times 10^{\wedge} 3 / \mathrm{ml}\right)$ and elevated C-reactive protein 
value $(95.5 \mathrm{mg} / \mathrm{dl})$, serum chemistry values were within the normal range (Table 1) and the estimated glomerular filtration rate (EGF) was $131 \mathrm{ml} / \mathrm{min}$; EKG (Figure 2A) and echocardiography revealed normal cardiac function and morphology. Because of the clinical suspect of tuberculosis, the patient underwent biopsy of the major chest and nucleic acid amplification confirmed the diagnosis of tuberculosis. Accordingly, the anti-tuberculosis therapy was initiated, with rifampicin $600 \mathrm{mg} /$ day, isoniazid $200 \mathrm{mg} /$ day, ethambutol 1200 $\mathrm{mg} /$ day, pyrazinamide $1500 \mathrm{mg} /$ day. To achieve a total pain control, Pregabalin $150 \mathrm{mg} /$ day, Tapentadol $100 \mathrm{mg} /$ day, and Paracetamol 2 $\mathrm{g} /$ day were also prescribed.

About 3 weeks later, during a routine check, a pulse heart rate of 35 bpm was unveiled. An EKG was promptly performed and a 1st degree A$\mathrm{V}$ block with a PR interval duration of $480 \mathrm{msec}$ was detected (Figure 2B). Because of the lack of serum electrolyte abnormalities or detectable underlying cardiac diseases, we suspected that the AV block might be a drug side effect. We then performed a careful medication review, analyzing potential cardiac side effects or drug-to-drug significant interactions. No drugs were deemed likely to be associated to bradswelling. Acid fast bacilli (AFB) microscopy, mycobacterial culture,

Table 1. Serum chemistry on admission.

\begin{tabular}{lcc} 
& & Normal values \\
$\mathrm{Na}^{+}(\mathrm{mmol} / \mathrm{L})$ & 136 & $136-145$ \\
\hline $\mathrm{K}^{+}(\mathrm{mmol} / \mathrm{l})$ & 3.9 & $3.5-5.1$ \\
\hline $\mathrm{Cl}^{-}(\mathrm{mmol} / \mathrm{L})$ & 96 & $98-107$ \\
\hline Total Ca++ $(\mathrm{mg} / \mathrm{dl})$ & 10 & $8.5-10.5$ \\
\hline Glucose $(\mathrm{mg} / \mathrm{dl})$ & 83 & $70-110$ \\
\hline Urea $(\mathrm{mg} / \mathrm{dl})$ & 28 & $18-55$ \\
\hline Creatinine $(\mathrm{mg} / \mathrm{dl})$ & 0.8 & $0.7-1.2$ \\
\hline Proteins $(\mathrm{g} / \mathrm{dl})$ & 7.3 & $6.4-8.3$ \\
\hline Albumin $(\mathrm{g} / \mathrm{dl})$ & 4 & $3.2-4.6$ \\
\hline Total bilirubin $(\mathrm{mg} / \mathrm{dl})$ & 0.8 & $0.2-1.2$ \\
\hline AST $(\mathrm{U} / \mathrm{l})$ & 15 & $0-34$ \\
\hline ALT $(\mathrm{U} / \mathrm{l})$ & 11 & $0-55$ \\
\hline LDH $(\mathrm{U} / \mathrm{l})$ & 194 & $125-243$ \\
\hline CK $(\mathrm{U} / \mathrm{l})$ & 104 & $30-200$ \\
\hline CRP $(\mathrm{mg} / \mathrm{l})$ & 95.5 & $0-0.50$ \\
\hline
\end{tabular}
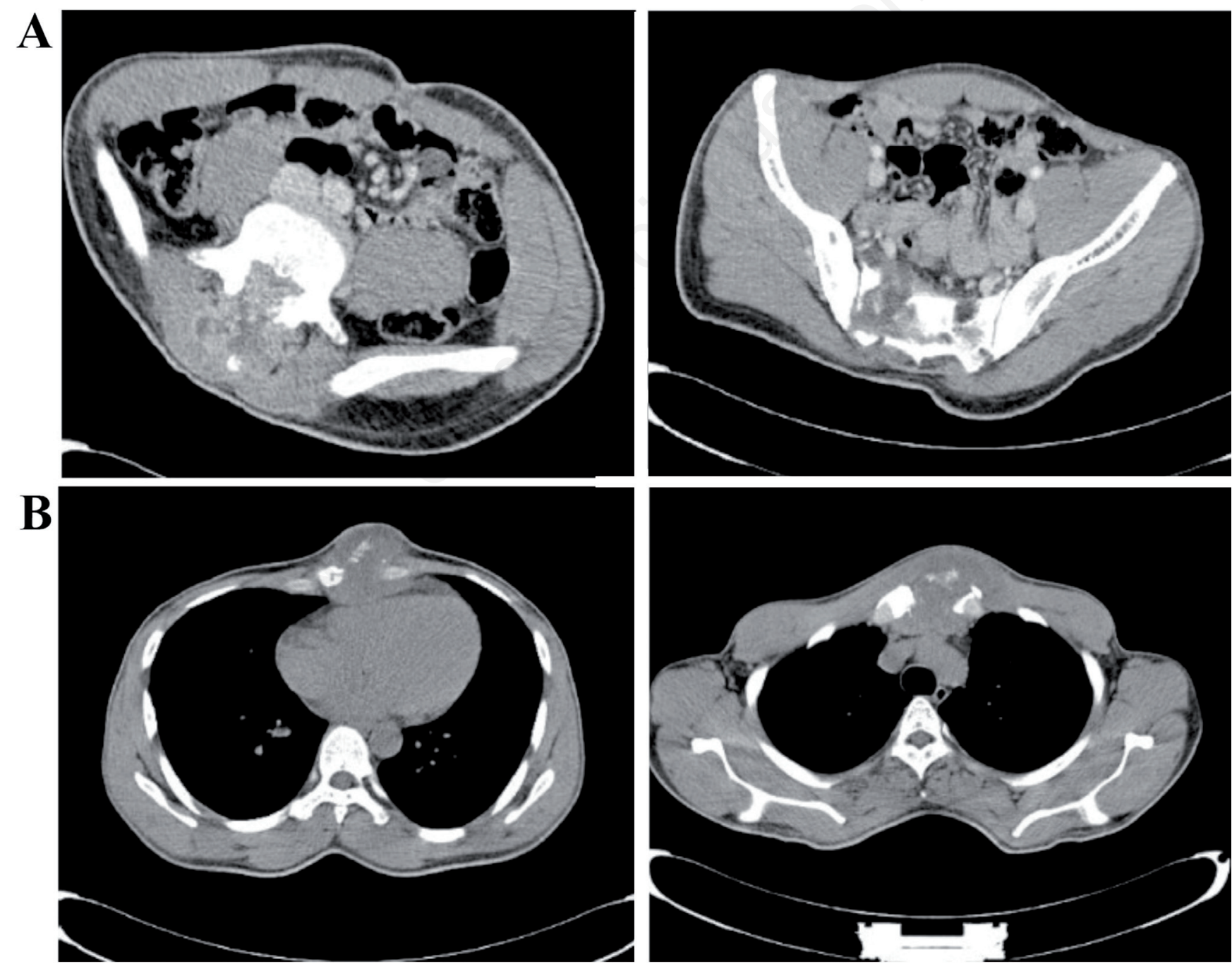

Figure 1. CT scan showing a mass involving the spinal cord (L4-L5/L5-S1) and the sacral bone (A) and a voluminous mass involving the sternum (B). 
yarrhythmias, with the exception of pregabalin. Therefore, pregabalin was discontinued. Consistent with this hypothesis, the EKG, recorded the following day, revealed normal AV conduction (Figure 2C). According to the principles of the good clinical practice, the adverse event was reported to AIFA through the qualified person responsible for pharmacovigilance at our institution.

\section{Discussion}

Atrioventricular blocks following pregabalin therapy have been previously reported only in patients with underlying cardiovascular diseases or impaired kidney function. In the current case report, we describe for the first time the occurrence of a 1 st degree A-V block during treatment with pregabalin in a young patient with normal kidney function and free of cardiovascular diseases.
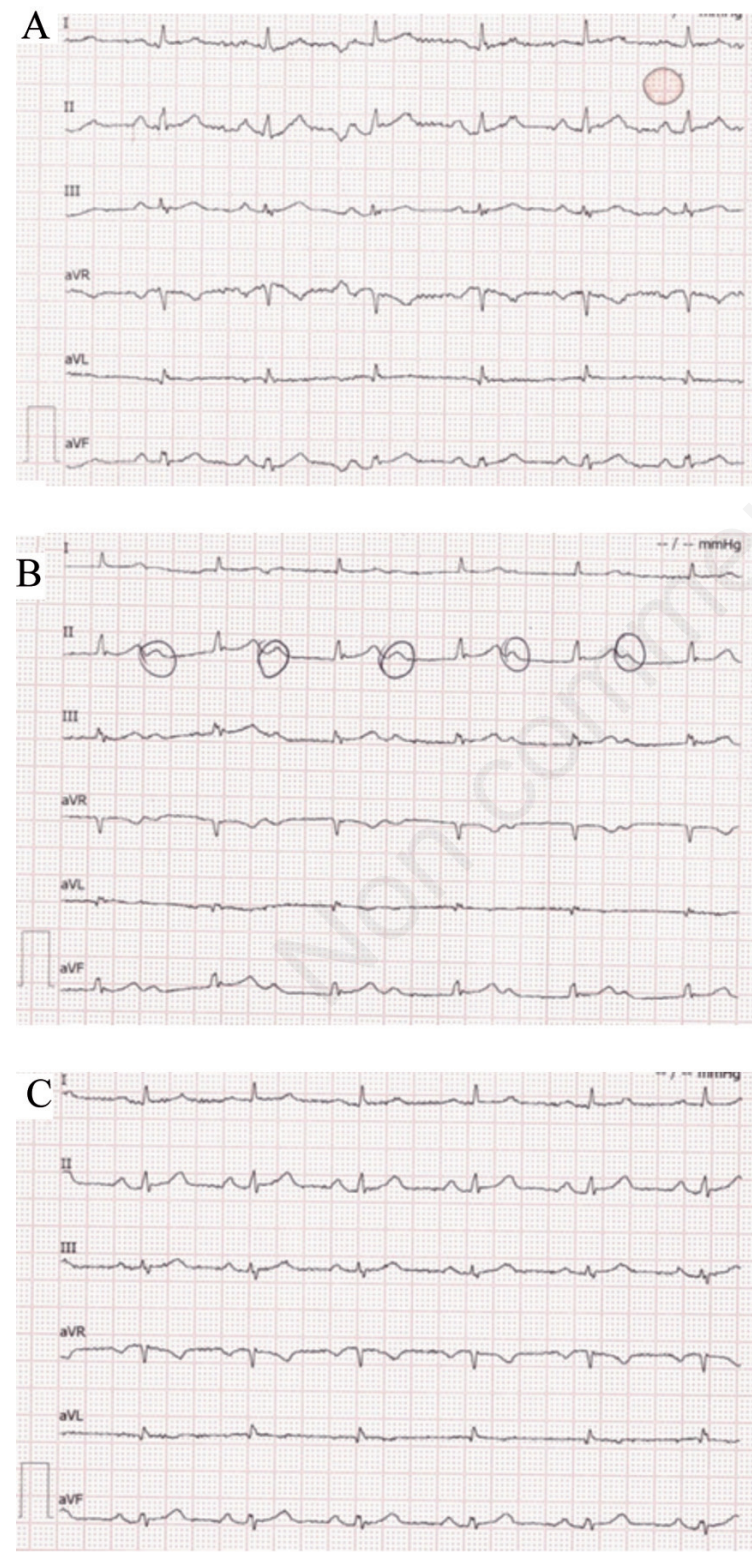

To evaluate the plausible association between the $1^{\text {st }}$ degree $\mathrm{A}-\mathrm{V}$ block we detected in the patient and the pregabalin administration, we used a validated adverse drug reaction scale [2]. Such an approach is currently used to estimate the probability of adverse drug reactions. Accordingly, our test drug scored as 'probable cause' (score 6) of the side effect we observed [2].

In the serum, pregabalin is not protein bound and its plasma half-life is approximately 6 hours and the maximum recommended dosage is 600 $\mathrm{mg} /$ day. The drug does not undergo hepatic metabolism and is cleared by renal excretion. Therefore, to maintain the drug levels within the normal therapeutic range, the dosage should be adjusted only for patients with impaired renal function, i.e. with an EGF lower than $60 \mathrm{~mL} / \mathrm{min}$. Since our patient had a normal renal clearance (EGF $131 \mathrm{ml} / \mathrm{min}$ ) and we used a dosage of $150 \mathrm{mg} /$ day, the current case report strikingly shows the possibility of cardiovascular adverse reactions with pregabalin also when the drug is used well within the recommended therapeutic range dosage.
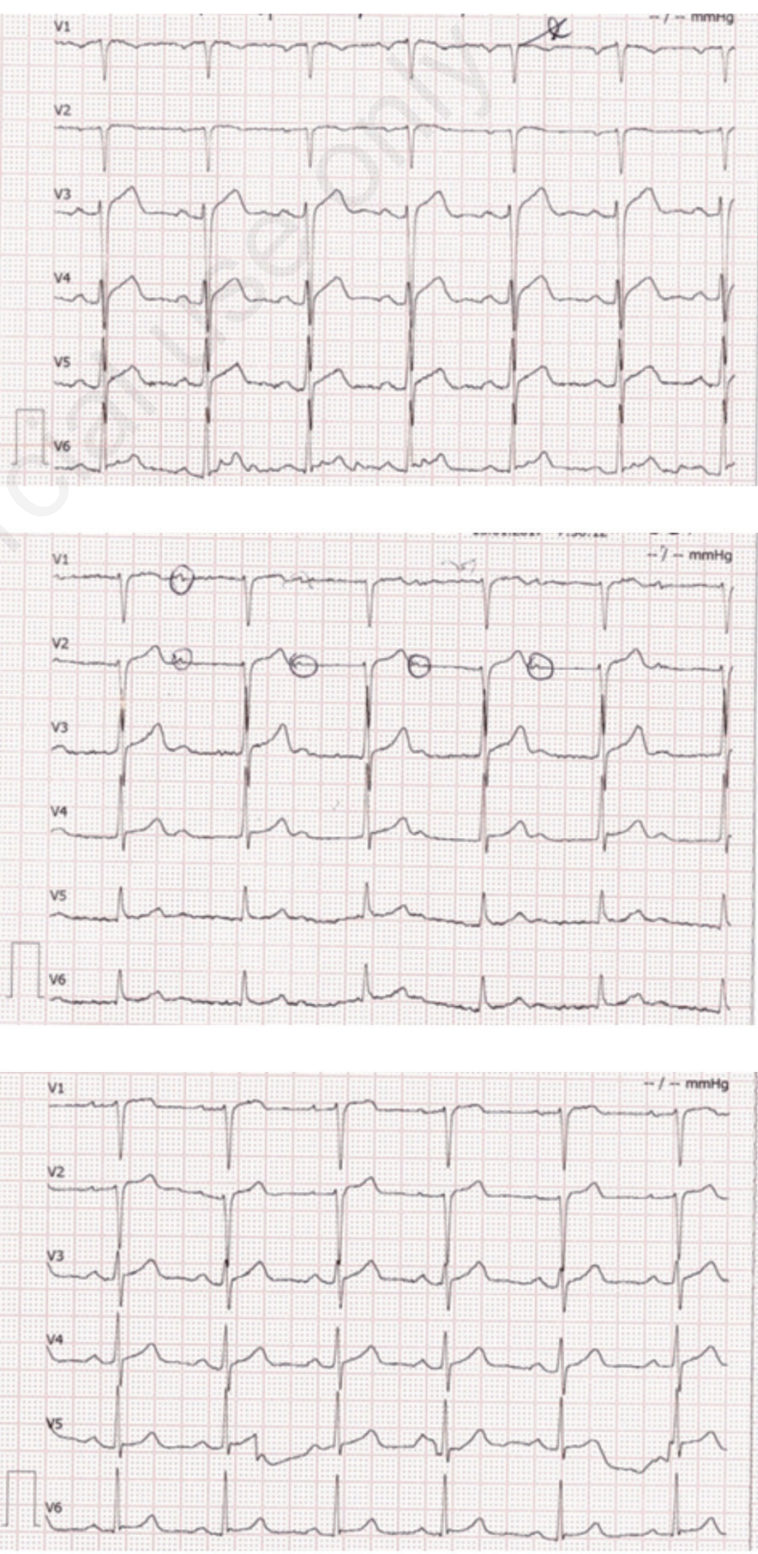

Figure 2. EKG performed on the patient. A) at admission to the medical unit (sinus rhythm); B) during the treatment with pregabalin ( $1^{\text {st }}$ degree AV block, PR = $\left.480 \mathrm{msec}\right)$; C) $24 \mathrm{~h}$ after pregabalin discontinuation (sinus rhythm). 


\section{Cardiac side effects associated with pregabalin}

L-type calcium channels are main regulators of calcium influx into cardiomyocytes, playing a key role in the "excitation-contraction coupling" [3]. For instance, non-dihydropyridine calcium blockers, such as verapamil, are selective for the blockade of L-type calcium channels and increase both functional and effective refractory period of the A-V node in a dose-dependent fashion. Pregabalin is a selective, high-affinity ligand for the $\alpha 2-\delta$ subunit of voltage-gated L-type calcium channels [3-4]. Therefore, cardiac side effects related to pregabalin use might be due to the interferences of the drug with the calcium channels of the cardiomyocytes. We suppose that the AV block we observed, occurred as the result of pregabalin effect on L-type calcium channels of the heart, mainly in the region of $\mathrm{AV}$ node. In particular, the drug might have reduced the calcium influx into the nerve terminals and decreased the release of neurotransmitters, resulting in an inhibitory modulation of neuronal excitability.

A case of a complete AV block has been previously reported in a patient with dilatative cardiomyopathy. In that patient, the AV block was probably aggravated by the impairment of kidney function that might likely have reduced pregabalin clearance resulting in a drug overdose [5]. A case of incomplete AV block has been also reported in a patient with normal renal clearance but with history of myocardial infarction and stroke and treated with pregabalin for neuropathic pain. In the latter patient, the PR interval was slightly longer than normal (240 msec) [6]. In the case we describe, in addition to the lack of kidney and heart disease of the patient, the elongation of the PR interval till the level of $480 \mathrm{msec}$ is particularly surprising and alarming.

A few other reports are present in literature regarding additional cardiac side effects of pregabalin [7-9]. However, all of them reported cases of acute decompensation in patients with advanced or early stage chronic heart failure, a disease that impact heavily on patient survival and quality of life [10-12]. They suggested that calcium-channel blocking induced by pregabalin triggered a deleterious effect on ventricles especially in patients with left ventricular systolic dysfunction. In those circumstances, pregabalin likely worked as a trigger, unleashing mechanisms underlying the heart failure condition [13].

Thus, the fact that in the current case the A-V block occurred in a young patient without underlying kidney or cardiac diseases and well within the therapeutic dosage of pregabalin is particularly relevant.

Nevertheless, we have been wondering whether the response to pregabalin we observed was peculiar because of the black race of the patient. Therefore, we searched the literature and did not detect any information regarding differences among races in the expression of the calcium channel $\alpha 2-\delta$ subunit protein isoforms, target of the pregabalin action. However, a few comments against the possible link between AV block and black race can be drawn.

Firstly, pregabalin renal clearance is not different in Blacks compared to Caucasians or Hispanic [14]. Secondly, no differences in the therapeutic effects of the drug due to races have been reported. Finally, if any difference is present between black people and other races, a reduction, rather than an increase, in the frequency or severity of the most common adverse events has been reported in the black population [15]. Ultimately, there is no information on difference in calcium channel protein isoform frequency or distribution in black versus other races and the available data suggest that the adverse reactions to pregabalin do not appear to hit preferentially black people.

\section{Conclusions}

We describe for the first time the occurrence of 1 st degree AV block due to regular pregabalin treatment in a young patient without cardiac or kidney diseases. The current case highlights the possibility of cardiac adverse reaction to pregabalin even when the drug is used well within its therapeutic range and in patients without underlying heart or renal diseases. Since we identified the AV block by chance, being the patient in the ward, we conjecture that similar events might occur even frequently, but they go undetected or misinterpreted and undertreated. Therefore, we suggest that monitoring of AV node conduction by EKG should be routinely considered before and during treatment with pregabalin.

\section{References}

1. Gajraj NM. Pregabalin for Pain management. Pain Pract 2005;5:95102.

2. Naranjo CA, Busto U, Sellers EM et al. A method for estimating the probability of adverse drug reactions. Clin Pharmacol Ther 1981;30:239-45.

3. Alp R, Citil M, Uzun M, et al. Effects of therapeutic doses of pregabalin on QTc interval in conscious rabbits. Eur Rev Med Pharmacol Sc 2008;12:223-8.

4. Yada H, Murata M, Shimoda K et al. Dominant negative suppression of Rad leads to QT prolongation and causes ventricular arrhythmias via modulation of L-type $\mathrm{Ca} 2+$ channels in the heart. Circ Res 2007;101:69-77.

5. Aksakal E, Bakirci EM, Emet M, Uzkeser M. Complete atrioventricular block due to overdose of pregabalin. Am J Emerg Med 2012;30: 2101.el-4.

6. Scarano V, Casillo R, Bertogliatti S, Orlando V. [Incomplete atrioventricular block in a patient on pregabalin therapy].[Article in Italian]. Recenti Prog Med 2013;104:574-6.

7. Murphy M, Mockler M, Ryder M, et al. Decompensation of chronic heart failure associated with pregabalin in patients with neuropathic pain. J Card Fail 2007;13:227-9.

8. Fong T, Lee AJ. Pregabalin-associated heart failure decompensation in a patient with a history of stage I heart failure. Ann Pharmacother 2014;48:1077-81.

9. Erdoğan G, Ceyhan D, Güleç S. Possible heart failure associated with pregabalin use: case report. Agri 2011;23:80-3.

10. Bossone E, Limongelli G, Malizia G, et al. The T.O.S.CA. Project: research, education and care. Monaldi Arch Chest Dis 2011;76:198-203.

11. Marra AM, Arcopinto M, Bobbio E, et al. An unusual case of dilated cardiomyopathy associated with partial hypopituitarism. Intern Emerg Med 2012;7(Suppl 2):85-7.

12. Arcopinto M, Salzano A, Bossone E, et al. Multiple hormone deficiencies in chronic heart failure. Int J Cardiol 2015;184:421-3.

13. Page RL, Cantu M, Lindenfeld J, et al. Possible heart failure exacerbation associated with pregabalin: case discussion and literature review. J Cardiovasc Med 2008;9:922-5.

14. Bockbrader HN, Burger P, Knapp L, Corrigan BW. Population pharmacokinetics of pregabalin in healthy subjects and patients with chronic pain or partial seizures. Epilepsia 2011;52:248-57.

15. Freynhagen R, Serpell M, Emir B, et al. A comprehensive drug safety evaluation of pregabalin in peripheral neuropathic pain. Pain Prac. 2015;15:47-57. 